\title{
Development of Acoustic Emission Technology for Condition \\ Monitoring and Diagnosis of Rotating Machines; Bearings, Pumps, \\ Gearboxes, Engines and Rotating Structures
}

\author{
D. $\mathrm{Mba}^{1} \&$ Raj B.K.N. Rao ${ }^{2}$ \\ ${ }^{1}$ School of Engineering, Cranfield University, UK \\ ${ }^{2}$ COMADEM International, UK
}

\begin{abstract}
One of the earliest documented applications of Acoustic Emission Technology (AET) to rotating machinery monitoring was in the late 1960s. Since then there has been an explosion in research and application based studies covering bearings, pumps, gearboxes, engines and rotating structures. This paper presents a comprehensive and critical review to date on the application of Acoustic Emission Technology to condition monitoring and diagnostics of rotating machinery.
\end{abstract}

Keywords: Acoustic Emission, condition monitoring, machine diagnosis, rotating machines.

\section{Introduction}

Acoustic emissions (AE) are defined as transient elastic waves generated from a rapid release of strain energy caused by a deformation or damage within or on the surface of a material $[1,2,3]$. In the application to rotating machinery monitoring, AE are defined as transient elastic waves generated by the interaction of two media in relative 
motion. Sources of AE in rotating machinery include impacting, cyclic fatigue, friction, turbulence, material loss, cavitation, leakage, etc. For instance, the interaction of surface asperities and impingement of the bearing rollers over a defect on an outer race will result in the generation of acoustic emission. These emissions propagate on the surface of the material as Rayleigh waves and the displacement of these waves is measured with an AE sensor. Rayleigh waves are a combination of longitudinal and transverse waves [4]. It should be noted that surface defects such as cracks and scratches attenuate rayleigh waves, in addition, the surface finish of metals can also influence attenuation [4].

Judicious application of well-tried and tested acoustic emission technology can provide powerful diagnostic capabilities, which are safe, efficient and cost-effective. This paper reviews the research and development activities that are being pursued in the following subject areas; bearings (roller and hydrodynamic), gearboxes, pumps, machinery and mechanical seals.

Acoustic emission (AE) was originally developed for non-destructive testing of static structures, however, over the last 35 years its application has been extended to health monitoring of rotating machines, including bearings, gearboxes, pumps, etc. It offers the advantage of earlier defect/failure detection in comparison to vibration analysis due to the increased sensitivity offered by AE. However, limitations in the successful application of $\mathrm{AE}$ technique for monitoring the performance of a wide range of rotating machinery have been partly due to the difficulty in processing, interpreting and classifying the intelligent information from the acquired data. The main drawback with the application of the $\mathrm{AE}$ technique is the attenuation of the signal and as such 
the AE sensor has to be close to its source. However, it is often practical to place the $\mathrm{AE}$ sensor on the non-rotating member of the machine, such as the bearing or gear casing. Therefore, the AE signal originating from the defective component will suffer severe attenuation, and reflections, before reaching the sensor.

AE covers a wide frequency range $(100 \mathrm{kHz}$ to $1 \mathrm{MHz})$ and time domain waveforms associated with $\mathrm{AE}$ are of two types; burst and continuous. A continuous type $\mathrm{AE}$ refers to a waveform where transient bursts are not discernible [5]. Both waveform types are associated with rotating machinery, for instance, a continuous type emission may be as a result of turbulent fluid flow within a peep while a burst type could be associated with transient rolling action of meshing bears. On rotating machinery typical background operational noise is of a continuous type. Traditionally the most commonly measured AE parameters for diagnosis are amplitude, r.m.s, energy, kurtosis, crest factor, counts and events [3]. Observations of the frequency spectrum, whilst informative for traditional non-destructive evaluation, have only found limited success in machinery monitoring. This is primarily due to the broad frequencies associated with the sources of generation of AE in rotating machinery. For example, the transient impulse associated with the breakage of contacting surface asperities experiencing relative motion will excite a broad frequency range.

\section{Acoustic Emission and bearing defect diagnosis}

From the moment bearings leave the factory, they encounter many harsh environmental hazards, which in turn induce a number of failure modes. It is well 
known how these failure modes reduce the life expectancy of the bearings. Some of the events responsible for the bearing failures include; incorrect applications, poor maintenance, poor lubrication, overload, over-speed, misalignment, imbalance, harsh environmental conditions (temp/humidity/dust/dirt/altitude), etc. Bearing failure modes include; friction/wear processes producing flaking, brinelling, fluting, spalling, pitting, seizure, etc. All these modes are known sources of AE. However, the most widely employed technique for condition monitoring and diagnostics of bearings is vibration monitoring. This method has been successful where the energy from other components (shaft, gears, etc) does not overwhelm the lower energy content from the defect bearing. In addition, by the time a significant change in vibration has been observed the remaining operational or useful life of the bearing is very short. This is where the AE technology offers a significant advantage. The formation of subsurface cracks due to the Hertzian contact stress induced by the rolling action of the bearing elements in contact with the inner and outer races, and, the rubbing between damaged mating surfaces within the bearing will generate acoustic emission activity. Other reasons for the generation of $\mathrm{AE}$ include the breakdown of the oil film, foreign matter in the lubricating medium and excessive temperatures. It must be noted that the propagation of the acoustic emission is affected by material microstructure, nonhomogeneities, geometrical arrangement of free surfaces, loading conditions and number of component interfaces. Almost all research on the application of AE to bearing defect analysis has been undertaken on experimental test-rigs specifically designed to reduce AE background noise.

Catlin [6] reported AE activity from bearing defects were attributed to four main factors including numerous transient and random AE signals associated with bearing 
defects. Furthermore, it was stated that the signals detected in the AE frequency range represented bearing defects rather than other defects such as imbalance, misalignment, looseness, shaft bending as well as the other major structural component resonance's. In addition, Catlin noted that high frequency AE signatures attenuate rapidly; therefore, if the transducer was placed close to the bearing, it was possible to detect the high frequency content induced mainly by the bearing fault since signatures originating from other machine components are highly attenuated upon reaching the sensor. Balerston [7] published the first document that applied the AE technology to identification of artificially seeded defects in rolling element bearings. Interestingly this is probably one of the earliest applications of AE to monitoring bearings. Defect simulated included outer and inner race defects, ball defects and lack of lubrication. Balerston compared vibrations in the audible range, resonant range and $\mathrm{AE}$, commenting on the advantages that monitoring of the resonant frequency range offered over the audible vibration range. The resonant technique involved measurement of bearing component natural frequencies initiated by shock excitation associated with minor structural irregularities. These resonant frequencies are a function of the mass configuration and type of material involved. The frequencies and amplitudes at resonance are much higher than bearing element rotations, so they are ideal under conditions of high background noise. Moreover, the resonant frequencies are independent of rotational speed, however, their amplitudes will vary directly with rotational speed, as will the impact energy. Resonant frequencies can be as high as $300 \mathrm{kHz}$ for ball rollers, and up to $140 \mathrm{kHz}$ for the inner race, depending on the mode of vibration [7]. Balderstone suggested that the 'free' resonant frequencies of the individual components were not changed significantly after assembly, though, the assembly created a damping effect. Furthermore, it was suggested that because of the 
interaction between the components of a bearing, a defect in any component would cause resonant frequency ringing in all components, making interpretation difficult. Moreover, at low rotational speeds the impact energy generated will be very low and this might explain why there have been limited applications of this technique to lowspeed bearings. The principal of the shock pulse meter (SPM) is similar to the resonant technique as both respond to minute transient pressure waves generated from fault impacts in regions of contact, however, the SPM resonates itself.

Balerston noted that two types of AE signatures were observed during experimental testing; burst type emissions associated with the seeded defects on the inner, outer race and ball element. Continuous type $\mathrm{AE}$ signatures were noted when the bearing was run dry (starved of lubrication). In one particular bearing defect simulations (dry run) AE counts were noted to increase prior to bearing failure. In summary Balerston stated that the resonant frequency technique was very successful and it offered a direct correlation between defect severity and increase in amplitude level of the resonant frequencies, though it was concluded the $\mathrm{AE}$ technique would become important with development of sensors. This was the earliest assessment on the application of AE to bearing monitoring.

About 10 years after Balerston, Rogers [8] utilised the AE technique for monitoring slow rotating anti-friction slew bearings on cranes employed for gas production and obtained some encouraging results compared to vibration monitoring techniques. Rubbing of the crack faces, grinding of the metal fragments in the bearing and impacts between the rolling elements and the damaged parts in the loaded zone were identified as sources of detectable AE signatures. Roger stated "because of the slow 
rotational speed of the crane, application of conventional vibration analysis (0$20 \mathrm{KHz})$ was of limited value for on-line condition monitoring". AE resonant transducers between $100 \mathrm{kHz}$ to $300 \mathrm{kHz}$ were found to be informative for on-line monitoring of bearings using kurtosis at different frequency bands.

Yoshioka and Fujiwara [9, 10] have shown that AE parameters identified bearing defects before they appeared in the vibration acceleration range. In addition, sources of AE generation were identified during fatigue life tests on thrust loaded ball bearings. Hawman and Galinaitis [11] reinforced Yoshioka's observation that AE provided earlier detection of bearing faults than vibration analysis and noted that diagnosis of defect bearings was accomplished due to modulation of high frequency AE bursts at the outer race defect frequency. Hawman and Galinaitis placed the AE receiving sensor directly onto the bearing outer race. The modulation of $\mathrm{AE}$ signatures at bearing defect frequencies has also been observed by other researchers $[12,13]$. In addition, Bagnoli et al [14] investigated demodulation of AE signatures at the defect rotational frequency (outer race) of a bearing. It was noted that when the defect was absent, the periodicity of the passage of the balls beneath the load could be readily identified by observing the frequency spectrum of demodulated AE signatures, however, it was reported that the AE intensity was less without the defect present. There was no mention of trigger levels employed, load applied on the test bearing, method of attaching the transducers to the rig nor any information on background noise.

Tandon and Nakra [15] investigated AE counts and peak amplitudes for an outer race defect using a resonant type transducer. It was concluded that AE counts increased 
with increasing load and rotational speed. However, it was observed that AE counts could only be used for defect detection when the defect was less than $250 \mu \mathrm{m}$ in diameter, though AE peak amplitude provided an indication of defects irrespective of the defect size. Loads applied ranged from 8 to $50 \%$ of the bearing static load rating. Choudhary and Tandon [16] employed AE for bearing defect identification on various sized bearings and rotational speeds ranging from 500 to $1500 \mathrm{rpm}$. It was observed that $\mathrm{AE}$ counts were low for undamaged bearings. In addition, it was observed that AE counts increased with increasing speed for damaged and undamaged bearings whilst an increase in load did not result in any significant changes in AE counts for both damaged and undamaged bearings.

Tan [17] used a variation of the standard AE count parameter for diagnosis of different sized ball bearings. In addition to the difficulty of selecting the most appropriate threshold level for standard AE counts, Tan cited a couple of other drawbacks with the conventional AE count technique. This included dependence of the count value on the signal frequency. Secondly, it was commented that the count rate was indirectly dependent upon the amplitude of the AE pulses. Tan's variation to the standard AE counts technique involved computing the accumulated area under the amplitude-time curve of the AE waveform over a specified time period. This was accomplished by setting four trigger levels with amplitude multiples of 1, 2, 4 and 8, and calculating the area under the amplitude-time AE waveform. The final count assigned was weighted by the multiple of the amplitude ratio between these levels. It was concluded that the 'new' count rates increased exponentially with increasing defect sizes and increasing rotational speed. The dependence of AE counts on threshold levels was also noted by Huguet et al [18] during investigations on the use 
of AE for identifying damage modes in specific materials, in this instance, a trigger level of $10 \%$ of the maximum amplitude was employed.

Yoshioka et al [19] undertook an investigation of vibration and AE on naturally fatigued deep groove ball bearings (bore diameter 20mm). By removing the groove on the inner race Yoshioka claimed the stresses in the area of contact were increased and this accelerated fatigue failure. Vibration r.m.s levels were recorded continuously through the fatigue tests which lasted approximately 130 hours. The presence of spalls on the inner race resulted in a rapid increase in vibration r.m.s levels. However, AE (counts per minute) showed a steadily increasing value at least 5 hours before the observed rapid increase in vibration. A total of sixteen fatigue tests were undertaken and the authors commented that they could predict the appearance of a spall by observing the AE response.

Whilst AE counts maybe highlight changes in machine state it will not be able to identify the origins of defect, e.g., outer race. The successful use of AE counts for bearing diagnosis is dependent on the particular investigation, and, the method of determining the trigger level is at the discretion of the investigator. Moreover, it has been shown that $\mathrm{AE}$ counts are sensitive to the level and grade of lubricant within the bearing, adding to the complexity of this measure. Morhain and Mba [20] undertook an investigation to ascertain the most appropriate threshold level for $\mathrm{AE}$ count diagnosis in rolling element bearings. Results showed values of AE maximum amplitude did correlate with increasing speed but not with load and defect size. In addition, it has been shown that the relationship between bearing mechanical integrity and AE counts is independent of the chosen threshold level, although a threshold of at 
least $30 \%$ of the maximum amplitude for the lowest speed and load operating condition was advised. Furthermore Morhain and Mba commented that unlike the results reported by Tandon and Nakra [15] it was observed that AE counts could be used for defect size detection for lengths of up to $15 \mathrm{~mm}$ and widths of $1 \mathrm{~mm}$. In addition Morhain validated the observations of Choudhary and Tandon [16].

Kakishima et al [21] undertook a comparative experimental study on the assessment of Acoustic Emission and vibration for monitoring/detecting seeded defect simulations on the inner race of a roller and ball bearing. Defects were seeded with an electron discharge machine (EDM). Analysis of the AE was based on the spectrum of the enveloped $\mathrm{AE}$ signals. It was concluded that the threshold at which the $\mathrm{AE}$ technique was able to identify the defect was similar to vibration monitoring. Furthermore, for both $\mathrm{AE}$ and vibration, it was noted that an increase in defect size resulted in an increase of both $\mathrm{AE}$ and vibration levels on the envelope spectrum. Kaewkongka and $\mathrm{Au}$ [22] applied the $\mathrm{AE}$ technique on a rotor dynamic system onto which multiple defects were seeded, including a seeded defect on one of the bearings. It was shown the $\mathrm{AE}$ technique offered high sensitivity thereby allowing for discrimination of the multiple defect conditions. Success was based on minimum distance classifier. Schoess [23] presented results of an assessment of six different but relevant technologies for onboard monitoring of a railcar bearing. It was concluded that the AE technique offered the highest potential payoff. Schoess successfully evaluated the AE technique on an artificially damaged bearing on a railcar, concluding that the AE technique offered potential for condition based maintenance in the railroad industry. Price et al [24] assessed the vibration and AE techniques for monitoring rolling element bearing failures. Prices's experimental study focused on a 
4-ball machine from which AE activity, vibration, temperature, friction, etc were monitored as a function of time. It was noted that AE could detect distress within the test balls before the friction in the contact area increased noticeably. It was stated that increasing damaging results in increasing friction at the contact area.

Shiroishi et al [25] compared vibration and AE on seeded defective bearings operating at 1200rpm. Interestingly, Shiroishi defined the industry bearing failure criteria as being reached when a defect size reached $6.45 \mathrm{~mm}^{2}$; this value was cited from the published article of Hoeprich [26]. Defects of varying sizes were seeded on the outer and inner races. Shiroishi noted that the vibration offered better detection than the AE technique, and that the $\mathrm{AE}$ sensor was insensitive to inner race defects. In addition, on the parameters extracted from vibration and AE measurements, Shirosihi et al [25] noted that the peak ratio was the most reliable indicator of the presence of a localised defect with the r.m.s, kurtosis and crest factor showing decreasing reliability. The most significant observation from Shiroishi's investigation was the correlation between acceleration peak value and defect width. This correlation was first noted back in 1969 by Balerston [7] employing a monitoring system based on observations of bearing resonant frequencies. The most recent correlation between defect size and measuring parameter (AE) was noted by Al-Ghamdi et al [27,28]. A direct correlation between defect length (circumferential, along direction of rolling) and AE burst duration was observed under varying simulated defect cases. In addition, a correlation between the amplitude of the burst type AE signature (associated with the bearing defect) to the underlying continuous type emission was noted to increase with increasing defect width (perpendicular to rolling direction). 
$\mathrm{Li}$ et al [29] undertook bearing fatigue failure tests at 1600rpm and $167 \%$ of the rated radial load. To accelerate failure an initial defect was seeded with an electric discharge machine. Li commented that vibration and AE r.m.s increased with increasing defect severity. An adaptive scheme was proposed to predict conditions of defective bearings based on vibration and AE techniques. Vibha Bansal et al [30] applied AE as a quality control tool on reconditioned bearings. Bearings were tested at $3 \%$ of the load rating. It was noted that as the load increased there was little increase in the peak-to-peak amplitude level for standard (operational) and reconditioned bearings, however, the peak values of the reconditioned bearing was in some instances five-times that of a new bearing.

$\mathrm{Li}$ and $\mathrm{Li}$ [31] presented a pattern recognition technique for early detection of bearing faults using AE. Faults were seeded on an outer race, a roller and multiple outer race defects. It was noted that the occurrence of $\mathrm{AE}$ events at a rate equivalent to a bearing characteristic defect frequency was evidence of the presence of a localised defect. Li et al presented such a case with the seeded outer race defect but no results on the roller defect were presented. This was rather disappointing as $\mathrm{Li}$ and $\mathrm{Li}$ are the only investigators to attempt to diagnose roller defects with AE.

Sundt [32] detailed two cases where high frequency AE was applied to bearing defect detection. For the first case study high frequency signals associated with a hairline crack in the outer race the defect frequency were detectable above $100 \mathrm{kHz}$. This defect condition was not observed with vibration analysis. It was stated that the defect was at an early stage of development and the bearing clearances had not deviated from the normal operating condition; explaining why vibration monitoring was 
unsuccessful in this particular study. The second case study should the ability of the $\mathrm{AE}$ technology to detect the presence of foreign matter (sand) in the bearings of a pump unit. Sundt commented on the use of AE to defect defective bearings utilising race resonance for amplification noting that this could enhance detection sensitivity. However, it was stated that the mechanical 'Q' (dynamic magnification factor) of the race was an un-predictable function of the bearing type, housing constraint, etc. Furthermore, it was noted that race resonances could be excited by normal background noise. Also, similar readings could be obtained from a good bearing with a high 'Q' and a bad bearing with a low 'Q'. The difficulty with monitoring bearings at the element resonating range $(20-100 \mathrm{kHz})$ was also discussed by Barclay and Bannach [33]. It was noted that wavelengths of vibration at these frequencies are often comparable with the dimensions of parts in the bearing or bearing housing which may create standing waves with nodes and anti-nodes. The consequence of this makes sensor position critical. Barclay and Bannach [33] presented the Spectral Emitted Energy (SEE) method which combined the high frequency AE detection within 250 - $350 \mathrm{kHz}$ range with the enveloping technique. The source of AE activity was attributed to the metal-to-metal contact as a result of lubricating film breakdown. It was concluded that the SEE method was a viable technique for detecting rolling element bearing defects and compliments the present-day low frequency vibration.

Badi et al [34] investigated the condition of automotive gearbox bearings using Stress waves (also known as AE). These sensors were used on a bearing test rig with simulated faults. All the artificially seeded faults were identified by employing the stress wave sensor method. The sensors were easy to install \& needed simple signal processing to evaluate bearing faults. The only drawback was the sensors were bulky. 
Sturm et at [35] employed AE to investigate damage processes (pitting and mixed friction) of sliding and rolling element bearings under laboratory and field conditions. Analysis revealed that the amplitude behavior observed from the envelope analysis of the AE signals yielded essential information about the damage processes. Javed and Littlefair [36] presented some general aspects of the application of AE for detecting the early development of failures in rolling element bearings. Some results of the experimental investigation of the basic relationship between ball bearing failures and the resulting change in AE signal were presented. Neill et al [37] described the relative sensitivities of accelerometer and $\mathrm{AE}$ sensors to a range of defects and assessed their merits in an industrial environment, where ambient noise and/or other faults were highly influential. It was revealed that the $\mathrm{AE}$ signals preserve the impulsive nature of defect-element interactions, yielding characteristic harmonics of the defect frequencies in the spectrum. These harmonics distinguished bearing defects from other periodic faults induced by imbalance or misalignment occurring at the same frequency. Also, Neill concluded that the AE sensors were more sensitive to small defects.

Salvan et al [38] adopted a triangulation technique by employing two AE sensors with fuzzy neural networks on a high-speed post office mail sorting machinery, which contained a large number of bearings. The investigation was limited to the detection of a simpler source and the authors were unable to obtain precise location presumably due to incorrect parameters in the sound velocity equation and the use of an inefficient technique. Parikka et al [39] reported their findings on the operation of paper machines, which were equipped with a number of oil lubricated rolling bearings. They assessed information on the effects of higher or significantly lower than intended 
bearing loads on its service life, as the lubricant conditions or movement changes with time. It was commented that the possibility of using $\mathrm{AE}$ for monitoring critical operating situations of rolling bearings was very promising. Based on this investigation, a window-based diagnostic system (prototype) was developed. Morhain and Mba [40] investigated the application of standard AE characteristic parameters on lightly radially loaded bearing. An experimental test rig was designed to allow seeded defects on the inner/outer races. The test rig also produced high background AE noise providing a realistic test for fault diagnostics. It was concluded that irrespective of the high levels of background noise and low radial load (between 2 to $70 \%$ of the bearing rating), standard AE parameters provided adequate early indication of bearing defects. Fan et al presented data streaming technology for non-interrupted acquisition of $\mathrm{AE}$ waveforms. In addition, Fan re-iterated that modulation of the AE waveforms could identify the defective part (race, roller) within the bearing. Holroyd [42] detailed laboratory studies on rolling element bearings in which AE signals were processed in terms of their dynamic envelop (i.e, rectification and low pass filtering). Tests showed that the periodicity of the enveloped signal corresponded to a bearing defect frequency. A proprietary method of characterising the AE time waveform was proposed. Several successful applications of the proprietary method were also presented.

Finley [43] developed an incipient failure detection 'IFD' system based on high frequency AE's generated from shock pulses as a rolling element (ball) passes a defective race. A couple of industrial case studies were presented. Finley noted that the AE technology has been proven to be more effective than conventional low frequency sound and vibration measurements. 
Jamaludin et al [44] presented research findings on the lubrication monitoring of the low speed rolling element bearings (1rpm). A test rig was designed to simulate the real bearing used in real-life situations. Using a newly developed method called Pulse Injection Technique (PIT) the variation of lubricant amount in the low-speed bearing was successfully monitored. This technique was based on transmitting a Dirac pulse to the test bearing in operation via an $\mathrm{AE}$ sensor. The $\mathrm{AE}$ data was processed using a clustering technique based on AR coefficient to differentiate between properly and poorly lubricated bearings. The acoustic emission technique has also been employed by Miettinen et al $[45,46]$ and Holroyd [42] to monitor the lubricant condition in rolling element and plain bearings.

Whilst monitoring bearing degradation by $\mathrm{AE}$ and vibration analysis is relatively established at speeds above $600 \mathrm{rpm}$, at low-rotation speeds there are numerous difficulties with vibration monitoring which have been detailed [47, 48, 49, 50]. The difficulty of monitoring at low rotational speeds was summarised by Kuboyama [51].

Unlike vibration monitoring there has been considerable success in the development and application of AE to monitoring slow speed bearings. McFadden and Smith [52] explored the use of acoustic emission transducers for the monitoring of rolling element bearings at speeds varying from 10 to $1850 \mathrm{rpm}$. The sensors were placed on the bearing housing. A fault, simulated by a fine scratch on the inner raceway, formed the basis of this experiment. It was commented that the $\mathrm{AE}$ transducer, with a frequency response beyond $300 \mathrm{kHz}$, failed to perform as expected at the higher end of the rotational speed range (850 rpm) and was inferior to the conventional high- 
frequency accelerometer. However, at low rotational speeds $(10 \mathrm{rpm})$ the $\mathrm{AE}$ transducer appeared to respond to minute strains (local distortions) of the bearing housing caused by the concentrated loading of each ball in the bearing. These minute strains appeared as spurious spikes superimposed on the ball pass frequency. It was concluded that at low speeds with steady loads, base bending/strain of the bearing housing could enable the AE transducer to detect signatures from very small defects in rolling element bearings, while at higher speeds base bending appears as low frequency noise.

Smith [53] was involved in the experiment mentioned above and in a separate paper reiterated McFadden's [52] findings though puzzled at the behaviour of the AE sensor used, stating " the form of response of the AE sensor was puzzling since the transducer was responding to once-per-ball distorting in the casing at frequencies as low as $1 \mathrm{~Hz}$. AE transducers are not supposed to respond to frequencies as low as these".

Tavakoli [54] investigated the application of AE to needle bearings. Interestingly the rotational speed for this investigation was $80 \mathrm{rpm}$ which some might classify as a low speed application. Three simulations were undertaken; defect free fully lubricated, defect free un-lubricated and a condition in which two adjacent needle elements (rollers) were missing. The frequency domain characteristics of the AE r.m.s voltage were examined in relation to the simulated conditions. It was shown that the mean spectral density function of the r.m.s voltage distinguished all three simulations. It was also noted that the source of $\mathrm{AE}$ in bearings was attributed to friction and impacting. 
Holroyd [55] described in this application note the results of AE measurements on four heavily loaded roller bearings rotating at $60 \mathrm{rpm}$. The operation of these bearings in the slowly rotating machine was critical indeed. This case study clearly demonstrated the ability of this innovative and profitable technology to prevent secondary damage and to minimise production loss due to machine failures. Miettinen et al [56] described the use of the AE method in monitoring of faults in an extremely slowly rotating rolling bearing, whose rotational speed varied from 0.5 to 5 rpm. This investigation revealed that the AE measurement was very sensitive and the fault was easily identified under laboratory conditions. Jamaludin et al [57] reported the results of an investigation into the applicability of AE for detecting early stages of bearing damage at a rotational speed of 1.12rpm. A bearing test rig was used with seeded localised surface defects induced by spark erosion on the inner/outer races and on a roller element (which resembled pitting). The paper concluded that $\mathrm{AE}$ parameters such as, amplitude and energy provided valuable information on the condition of a particular low-speed rotating bearing.

Sato [58] investigated the use of AE to monitor low-speed bearing damage by simulating metal wipe in journal bearings at $5.5 \mathrm{rpm}$. It was observed that acoustic bursts were generated as a result of slight metallic contact and the amplitude of the waveform became larger with increasing metal wear. Sturm and Uhlemann [59] also investigated the application of AE to plain bearings, noting the instantaneous response of AE to the changes in the frictional state of hydrodynamic fluid film. 
Williams et al [60] noted that the majority of bearing diagnosis experiments were undertaken with seeded defects and as such undertook bearing experiments without seeded defects; in essence fatigue tests. The test bearings, roller and ball, were run at $6000 \mathrm{rpm}$ at $67 \%$ of the dynamic rated load, though some tests were undertaken at varying speed conditions. Vibration and AE techniques were compared and in one particular instance Williams stated that the AE sensor showed an increase 10 minutes after an increase in vibration. It was also noted that the AE sensor was unresponsive to outer race failures. This is rather surprising considering the number of publications confirming the ability of the AE technique to diagnose outer race defects.

The development of $\mathrm{AE}$ in bearing monitoring and fault diagnosis is the most established application of AE in rotating machinery and this is reflected in the number of commercially available systems in the market today. Needless to say, more detailed investigations are still required and there are opportunities for applying the $\mathrm{AE}$ technology for prognosis.

\section{Application of Acoustic Emission to monitoring gearboxes}

Whilst vibration analysis on gear fault diagnosis is well established, the application of $\mathrm{AE}$ to this field is still in its infancy. In addition, there are limited publications on application of AE to gear fault diagnosis. Irrespective of the numerous publications on the application of vibration analysis to monitoring gearboxes it still meets with great challenges that monitoring and diagnosis of gearboxes present. The AE technology offers a complimentary tool in this instance. 
Miyachika et al [61] presented a study on AE in bending fatigue test of spur gear teeth. Three different gears with common module, pressure angle and number of teeth were used. Two of the gears were case hardened to different case depths. These gears were made from SC415 steel with a face width of $10 \mathrm{~mm}$ whilst the second gear (face width of $8 \mathrm{~mm}$ ) was made from S45C steel without any case hardening. An AE sensor was fixed on the gear with a clamp arrangement. AE measurements such as frequency spectra, cumulative event count, event count rate and peak amplitude were recorded during the fatigue process under different tooth load conditions. In addition, crack length measurements were made. However, the type and characteristics of the sensor, the sampling rate employed and the loading frequency were not presented in this paper. During the fatigue test, it was observed that there was marked increase in $\mathrm{AE}$ cumulative event count and event count rate just before crack initiation for both case hardened gears. For the normalized gear, such an observation was not noted. It was also found that as the tooth load decreased, the number of cycles until the marked cumulative event count occurred increased. Miyachika drew the conclusion that the prediction of crack initiation using AE technique was possible for case hardened gear but difficult in the case of normalised gear.

Miyachika et al [62] extended their investigations to super-carburised gear material. The investigation was performed under the same test set and procedures as detailed above, with additional analysis techniques; AE cumulative energy count and wavelet transforms of AE signals. From the results, Miyachika concluded the prediction of crack initiation by means of AE method was possible for the various carburised gears tested. 
Wheitner et al [63] performed a series of gear tooth bending fatigue tests to verify the effectiveness of $\mathrm{AE}$ and system stiffness measurements for monitoring the crack initiation and propagation. The tests and instrumentation employed were to standards detailed in the Society of Automotive Engineers (SAE) gear geometry, testing procedure and fatigue test fixture. The $\mathrm{AE}$ senor had a resonant frequency of $300 \mathrm{kHz}$ was attached to the gear at the root of the tooth with super glue. The tooth stiffness measurements were done through an accelerometer mounted to the base of the fixture. The test gears were of various materials, surface finishes and surface treatments. All the testing was performed by applying sinusoidal load of $10 \mathrm{~Hz}$ and load ratio of 0.1 . A run-out life of $10^{6}$ cycles was employed for all the test cases. Wheitner noticed nonzero $\mathrm{AE}$ counts before the initiation point of the gear tooth root fatigue crack which was attributed to the background noise of the test machine. In general, AE activity increased with crack propagation and very rapidly at the failure point. All the test gears exhibited similar trends in stiffness measurements. At high load and low fatigue lives, crack propagation life contributed a significant proportion of the gear total life as compared to crack initiation life. Wheitner went further to conclude that both the $\mathrm{AE}$ and system stiffness measurements were effective in monitoring the cracking processes of the gear tooth. However, in most cases, AE activity was detected before the first change in stiffness compliance was registered.

Singh et al [64] explored an alternative AE technique to the more widely used vibration and debris monitoring methods for detection of gear tooth crack growth. He employed a single tooth bending machine with the load on the tooth varied sinusoidally at $40 \mathrm{~Hz}$ frequency. An AE sensor and accelerometer were mounted on a spur gear near to the loading tooth. The test terminated when the loaded tooth broke- 
off. Raw AE waveforms and fatigue cycles were recorded during the test. There was no information given on the type of gear, sensors, the applied load and the sampling rate used. The test revealed that AE detected the first sign of failure when the gear reached $90 \%$ of its final life. As the crack progressed, AE amplitude increased. During the final stage of gear tooth fracture, a significantly high amplitude AE burst was detected. On the other hand, the vibration level did not change significantly in the initial stage of crack initiation and propagation until the final stage of failure. Hence, Singh concluded that $\mathrm{AE}$ method offered an advantage over vibration monitoring techniques.

In order to study the practical aspects of sensor placement in a real life gearbox situation, Singh et al [64] performed an assessment of the transmissibility of an AE signal within a gearbox. The tests were performed with different torque levels using lead pencil breaks to simulate AE activity in the gearbox. This technique is known as the Nielsen source test. Firstly, various individual interfaces with varying torques were studied and quantified. Following that, Singh evaluated the total loss of strength of the AE signal across multiple interfaces and compared with the sum of losses obtained from individual interfaces. Several AE transmission paths were examined. From the results obtained, Singh et al concluded that the attenuation across the gearbox was an accumulation of losses across each individual interface within the transmission path and the optimum path of propagation will be the one with the smallest cumulative loss.

The investigations detailed earlier [61 to 64] have indicated that AE technique was able to detect bending fatigue failure. In addition, the AE technique was capable of 
detecting the fault condition in advance of the vibration monitoring technique. This conclusion is encouraging and motivating for $\mathrm{AE}$ technique to be the new condition monitoring tool. However, to ensure that this technique is robust, the defect detection capability on the other modes of gear failure; surface damage and fatigue, has to be explored.

Siores and Negro [65] explored several AE analysis techniques to correlate possible failure modes of a gearbox during its useful life through. The gearbox employed for the failure interrogation includes two gear sets (input and output), a DC shunt motor and a variable speed controller to alter the motor speed for the tests. The AE sensor employed was mounted on the gearbox casing and has a resonant frequency of 175 $\mathrm{kHz}$. Prior to the start of the test, the gearbox was allowed to wear-in at $1200 \mathrm{rpm}$ for four one-hour intervals at full load condition. Common gear failures such as excessive backlash, shaft misalignment, tooth breakage, scuffing and worn teeth were seeded on the test gears. All the seeded defect conditions were tested at 300 and $600 \mathrm{rpm}$ whilst AE parameters such as r.m.s, standard deviation and duration of AE were measured. Siores and Negro concluded that the monitored AE parameters exhibit identifying qualities for the respective failure modes.

Singh et al [66] performed two experiments to study the feasibility of applying AE to detect gear pitting. Both simulated and natural pits were used to evaluate this detection technique. The first experiment employed an UH1H generator drive offset quill which consisted of the driver, driven and idler gears. In this experiment, the idler gear contains the simulated pit of width and depth of $1.25 \mathrm{~mm}$. This pit was simulated by removing a thin strip of material from the pitch-line on one of the tooth of the idler 
gear by Electrical Discharge Machining (EDM) process. A resonant type AE sensor with a resonant frequency $280 \mathrm{kHz}$ and an accelerometer were mounted on the gearbox housing near the output shaft bearing. A tachometer was used as a trigger to ensure each cycle of the measurements started with the same idler tooth in contact. The test gearbox was first run with no pit on the idler gear and then replaced by the idler gear with simulated pits. AE and vibration data were recorded during the run. This procedure was repeated for several combinations of load and speed. From the test results, Singh concluded that both detection techniques were able to pick up the simulated defect but AE technique exhibited much greater signal to noise ratio (SNR). He also suggested that both detection techniques were unable to detect the simulated pit at extremely high speeds or unloaded conditions as the noise level increases whilst the amplitude of the defect signal arising from contact of the pitted region decreases.

Singh [66] performed the second experiment using a back-to-back gearbox to study the detectability of natural pits. Similar acquisition systems to the first experiment were employed with both the AE sensor and accelerometer mounted on the housing of the test gearbox. The input speed to the gearbox was 1775 rpm with an unknown torque loading. During the early stage of the test, there were no defects on the mating gear teeth surfaces and the signals (both $\mathrm{AE}$ and vibration) showed no significant peaks above the operational noise level. After 30 minutes of operation, pits started to develop on the pinion teeth and periodically occurring peaks were observed from the AE signals. A further 15 minutes run saw pitting on multiple teeth and the detected AE signals revealed more frequently occurred peaks above noise level. There was no visible peak noted for the accelerometer signal. During the test, AE sensor was also placed at the slave gearbox housing and bearing location between the two gearboxes 
to assess the detectability of the natural pits from the mentioned locations. Singh concluded that the AE sensor should be as close to the monitored part as possible in order to maximise the detection capability of pits using AE technique.

Raad et al [67] illustrated the application of AE monitoring technique for gear fault detection by employing an industrial gear rig. No information on the gear test rig, applied torque and speed were given in the paper. The experiment was performed above the rated load of the gears for two weeks until near breakage of two teeth. Various types of $\mathrm{AE}$ sensor (resonant and wide band) and accelerometers were mounted on the bearing. Measured signals were taken at regular intervals and visual inspection of gears was performed at end of each day. The recorded AE and vibration data were analysed using four different methodologies: visual comparison, Kurtosis, spectral density and envelope analysis. The visual comparison revealed that AE bursts appeared with spalling. However, these AE bursts disappeared after the defect was established. There was no clear indication from vibration signatures. The Kurtosis values were correlated to spalling defects after 3000 cycles. However, this method was unable to localise the spalling defect to individual tooth. The first sign of spalling observed from the vibration technique was at 5000 cycles. Using the spectral density analysis method, the increased in energy before and after the spall detection was common to both $\mathrm{AE}$ and vibration signals. In the final analysis of the $\mathrm{AE}$ and vibration signals, the spectrum of the squared envelope was used. Vibration technique was able to pick up the defect by displaying peaks at twice the shaft frequency. However, these peaks were not visible in the AE spectrum until the logarithm of the squared envelope was employed. The observed peaks occurred at the same frequency 
for both $\mathrm{AE}$ and vibration techniques. Raad concluded that this first evaluation of $\mathrm{AE}$ as condition monitoring tool is promising.

Sentoku [68] presented an investigation on tooth surface failure with AE measurements. A power circulating type gear testing machine was employed. The testing machine consisted of a pair of test and power return spur gears with a forced lubrication system that supply oil directly to the engaged teeth surfaces from the side of the gear pairs. It is important to note that the oil temperature was maintained constantly at $40 \pm 2{ }^{0} \mathrm{C}$. This eliminated the effect of oil film thickness on AE activity. An ultra-compact AE sensor of resonant frequency $350 \mathrm{kHz}$ was mounted on the gear wheel using screws. AE signature was transmitted from the sensor to the data acquisition card via a mercury slip ring. Strain gauge was also adhered to the tooth root to correlate the extracted AE parameters with tooth root strain waves. During the tests, the roughness of the gear teeth surfaces and pitting size were measured at regular intervals.

The first test was performed under applied stress of $960 \mathrm{MPa}$ and pinion speed of 992 rpm using hardened gears. From the results obtained, Sentoku observed no change in $\mathrm{AE}$ amplitude except the unevenness of $\mathrm{AE}$ wave lines were smaller with increasing number of cycles. At this stage of test, no surface damage was noted. Subsequently, Sentoku performed the second test using heat treated ground gears. During the early stage of the test, both $\mathrm{AE}$ amplitude and the pitting area ratio remained unchanged. However, when pitting on the three monitored gear teeth began, AE wave lines started to change. Subsequently, AE amplitudes increased with both the pitting area ratio and the numbers of cycle. Sentoku explained that the increase in AE amplitude was 
caused by friction due to increasing pitting. Similar observations were noted for AE energy. Hence, with the results obtained from the test, he drew conclusion that $\mathrm{AE}$ technique could detect gear teeth pitting.

Badi et al [69] performed an investigation on usage of AE and vibration monitoring techniques for condition monitoring of a typical drive-line. A test rig comprised of a drive and simple spur gearbox, loaded by pneumatically operated brake disk was employed to simulate the essential part of this drive-line. The rotating components were connected by flexible couplings and supported by bearing blocks. The rig was instrumented with both accelerometers and AE sensors at several locations along the drive line. However, Badi only reported the results from the sensor which gave the optimum location for fault detection. Seeded defects such as "blip” and "shaved” gear faults were introduced on the test gears to simulate scuffing and pitting defects on gear tooth. There was no further information on the testing procedures used in this experiment. Analysis techniques such as Crest Factor and Kurtosis were employed for both $\mathrm{AE}$ and vibration techniques. For the "blip" gear fault, both monitoring techniques were able to identify the defect through the analysis techniques employed. As for "shaved" gear fault, only the AE technique was able to detect the defect. Badi concluded that the analysis techniques used were ideally suited for identifying faults with impulsive nature. However, for a more comprehensive methodology, other analysis technique should be explored.

Tandon and Mata [70] performed seeded defect tests on spur gears using IAE gear lubricant testing machine to assess the fault detection capability of $\mathrm{AE}$ technique and make comparison with the more widely used vibration technique. Both hardened and 
ground spur gears were employed for the tests. The test gears were lubricated by a jet of oil. The AE sensor and accelerometer employed had a resonant frequency of 375 and $39 \mathrm{kHz}$ respectively. Both the AE and vibration signals were measured closed to the bearings of the test gearbox. All the tests were carried out at a single speed (1000 rpm) and varying load conditions (0 to $10 \mathrm{~kg}$ ). AE and vibration measurements were first taken for gears that have no seeded defect, which were treated as reference signals. Subsequently, a simulated pit of constant depth $(500 \mu m)$ and variable diameter (from 250 to $2200 \mu \mathrm{m}$ in incremental order) was introduced on a gear tooth pitch-line by spark erosion. From the tests, Tandon and Mata made these observations: (a) there was some increase in $\mathrm{AE}$ with increase in load. (b) $\mathrm{AE}$ parameters increased as the defect size (diameter of pit) increased. (c) AE (ring-down) counts showed slightly better results than other AE parameters measure. (d) AE technique detected the seeded defect at smaller size $(500 \mu \mathrm{m})$ compared to vibration technique $(1000 \mu \mathrm{m})$. (e) In general, the distribution of AE events, counts and peak amplitude became broader due to the presence of defect in gear.

Finley [43] presented an industrial case study on the application of an AE developed system (Incipeint Fault Detection) for gearbox monitoring. Al-Balushi and Samanta [71] introduced energy-based features extracted from AE signatures for monitoring and diagnosing gear faults. This feature, termed as energy index (EI), was defined as the square of the ratio of the r.m.s value for a segment of the signal to the overall r.m.s value of the entire signal. Various different forms of EI were derived and compared with existing statistical methods for early fault detection. Experiments were undertaken on a back-to-back spur gearbox. Three miniature ultra-sound transducers were implanted onto the rolling element bearing adjacent to the gear wheel for 
collection of AE data. A triggering system was used to ensure all the acquired data have identical starting locations on the gear. The tests were performed using brand new gears and terminated at the $40^{\text {th }}$ hours when the gear failed. AE signals were acquired for 1 revolution of the test gear at hourly interval. However, information such as the characteristics of the sensors, the applied load and the reason of the varying rotational speeds were undisclosed. Al-Balushi illustrated that the proposed EI and the various derived forms were able to locate the broken and pitting teeth more effectively than the traditional Kurtosis and Crest Factor methods. By employing the proposed analysis technique, the defective tooth was picked up in a helicopter gearbox.

In a separate report Al-Balushi and Samanta [72] presented a procedure for fault diagnosis of gears through wavelet transforms and ANN. The time domain AE signals of a rotating machine with normal and defective gears were processed through wavelet transform to decompose in terms of low frequency and high-frequency components. The extracted features from the wavelet transform were used as inputs to an ANN based diagnostic approach. The procedure was illustrated through the experimental AE signals of a gearbox.

Tan and Mba $[73,74]$ noted difficulties in identifying the location of a defective tooth during an experimental investigation. It was noted that the lubricant temperature had an influence on the levels of AE activity/strength during the gear mesh. This has far reaching consequences as it implies that whilst other researchers have stipulated the effect of load/speed on AE activity, the time of data acquisition, in effect the temperature of the lubricant will influence the levels of AE obtained. 
While exploring the applicability of $\mathrm{AE}$ technique to gear health diagnosis, Toutountzakis and Mba [75] made some interesting observations of AE activity due to misalignment and natural pitting. The test was performed on a back-to-back spur gearbox with the $\mathrm{AE}$ sensors placed on the pinion and bearing casing of the pinion shaft. The AE sensors used have a relative flat response in the region between 150 and $750 \mathrm{kHz}$. A silver contact air-cooled slip ring was employed to transmit the AE signal for further processing. AE parameters such as r.m.s and energy values were recorded during the tests. Prior to the test proper, AE measurements for defect free gears were firstly recorded. As the rotational speed increased, measured AE parameters increased for both AE sensor locations. Furthermore, Toutountzakis and Mba observed that change in speed resulted in changing AE parameters. During one of the tests, Toutountzakis et al noted increasing AE r.m.s (at pinion location) for 6 hours before the gearbox was paused for inspection. The results of the inspection revealed signs of pitting and scuffing which indicating a misalignment in the gearbox. The gearbox was reassembled and the test continued. An interesting observation was made: " $A$ reduction in $A E$ parameters was noted initially, but these values gradually increased to values which did not depart from the initial gradient of the increasing trend." Toutountzakis and Mba concluded that there is potential application of AE technique on gear health diagnostic.

Price et al [76] investigated the detection of severe sliding and pitting with AE. The experimental results presented were based on a 'four-ball machine' test-rig. It was observed that scuffing and pitting were easily detectable by observing changes in $\mathrm{AE}$ energy, principally due to changes in contact friction. More interestingly, Price noted 
changes in the frequency patterns of measure AE signals prior to pitting and stated that AE monitoring was capable of detecting wear events prior to either vibration monitoring or wear debris analysis. Building on this statement, very recently Tan et al $[77,78,79,80]$ presented results of an experimental investigation in which natural pitting of spur gears was allowed to occur. Throughout the test period, AE, vibration and spectrometric oil samples were monitored continuously in order to correlate and compare these techniques to natural life degradation of the gears. It was observed that the $\mathrm{AE}$ technique was more sensitive in detecting and monitoring pitting than either the vibration or Spectrometric Oil Analysis (SOA) techniques. It is concluded that as AE exhibited a direct relationship with pitting progression, it offered the opportunity for prognosis. From the results presented it was clearly evident that the AE monitoring indicator could be linearly correlated to the gearbox pitting rates for all torque conditions, with detection of onset of pitting as early as $8 \%$ of the pitted gear working face area. This offered much earlier diagnosis than vibration analysis where only after between 20 to $40 \%$ of pitted gear working face did this technique offer capability for defect identification. This near linear relationship between AE and pit progression offers great potential, and opportunities, for prognostics in rotating machinery.

Tan et al $[73,81,82,83]$ ascertained the AE source mechanism through a series of experimental programmes. These experimental programmes consisted of isothermal tests on undamaged gears to explore the effects of rotational speed and applied torque on AE levels. From the isothermal test results, it was observed that variation of the applied torque had a negligible effect on the AE r.m.s levels, similar to the negligible effect of load on film thickness under Elastohydrodynamic Lubrication (EHL) of non- 
conforming mating surfaces. It was noted that the variation in rotation speed had a more pronounced effect on AE r.m.s levels relative to the load. Tan concluded that the source of AE during gear mesh was asperity contact under rolling and sliding of the meshing gear teeth surfaces.

Although the development of $\mathrm{AE}$ in gear diagnosis is in its infancy, the papers reviewed have illustrated the potential and viability of $\mathrm{AE}$ becoming a useful diagnostic tool in condition monitoring of gears. However, more detailed investigations are required to ensure this technique is robust and applicable for operational gearboxes. This involves understanding the influences of operational variables on AE generation and investigating the effects of variable load conditions to monitoring with AE.

\section{Pumps and Acoustic Emission technology}

Pumps play a significant role in industrial plants and need continuous monitoring to minimise loss of production. Every pump manufacturer supplies characteristic curves for their equipment illustrating pump performance under given conditions. These curves demonstrate the inter-relationship between discharge capacities, pump head, power and operating efficiency. The ideal operating point for a pump is known as the best efficiency point, or BEP. This is the point where pump capacity and head pressure combine to provide the maximum efficiency of the pump. If the pump operates too far to the left or right of the BEP, not only may its efficiency be compromised, but it can also be subjected to increased wear, reducing operational life. 
Also, the pump manufacturer will undertake NPSH (Net Positive Suction Head) tests on supplied pumps; the significance of the latter is to determine the $3 \%$ drop in head at which serious cavitations will occur. Cavitation occurs when the absolute static pressure at some point within the pump falls below the saturated vapour pressure of the liquid. It causes a loss of pump efficiency and degradation of the mechanical integrity of the pump. It is generally accepted that the critical pressure for inception of cavitation is not constant and varies with operation fluid physical properties, the surface roughness of the hydraulic equipment, etc. In addition cavitation is known to begin long before the performance of the pump is affected [84].

This paper reviews the application of AE for condition monitoring of pumps. Prior to detailing some recent attempts at applying $\mathrm{AE}$ to pump health diagnosis, the investigation of McNulty and Pearsall, and, McNulty and Deeprose [84, 85] is worthy of mention. McNulty undertook high frequency measurements (up to $160 \mathrm{kHz}$ ) taken at the suction and discharge sides of the pump and detected incipient cavitation. However, it was noted that the success was dependent on the operational background noise levels. The results presented by McNulty relating NPSH to varying noise levels is of great interest, though undertaken at $40 \mathrm{KHz}$. It clearly relates the audible intensity and high frequency energy to the varying cavitation stages experienced by a pump as the head drops to the $3 \%$ level. It was noted [84] that during cavitation the high frequency noise increased. In separate paper McNulty [86] showed that minimum noise intensity levels of a pump were obtained at the BEP. Sources of noise were noted as turbulence, impeller and volute interactions and hydraulic interactions. 
Derakhshan et al [87] investigated the cavitation bubble collapse as a source of acoustic emission and commented that the high amplitude pressure pulse associated with bubble collapse generated AE. With the AE sensor was placed on the actual specimen experiencing cavitation Derkhshan observed increasing AE r.m.s levels with increased pressure of flow and cavitation. However, with the AE sensor mounted on the tank wall the reverse was observed, decreasing AE r.m.s levels with increasing pressure and cavitation. This was attributed to a visible bubble cloud that increased with pressure. It was commented that this cloud attenuated the AE signature prior to reaching the transducer on the wall casing. In addition to the high amplitude pressure pulse associated with cavitation, pressure pulses associated with centrifugal pumps have been detailed [88], these include wake flow from the impeller blade trailing edge, vortices generated by flow separation and recirculation. The influence of the latter on pump performance has been presented [89].

Neill et al [90, 91, 92] assessed the AE technology for detecting early cavitation. It was also noted that the collapse of cavitation bubbles was an impulsive event of the type that could generate AE. These transients cause very high local transient pressure that can damage the internal parts of pumps. It was observed that when the pump was under cavitation the AE operational background levels dropped in comparison to noncavitating conditions. To ensure a more direct transmission path between the fluid and the sensors, metal wave-guides were put into the venture tube wall at different locations. It is worth stating that prior to, and during cavitation, vibration measurements showed no significant change. In conclusion Neill stated that loss in NPSH before the 3\% drop-off criterion was detectable with AE and evidence of incipient cavitation was detectable in the higher frequency band (0.5 to $1 \mathrm{MHz})$. It is 
interesting to note that Neill [93] also successfully applied AE technology to detect the recirculation in a centrifugal pump. Recirculation is defined as a flow reversal at either the inlet or the discharge tips of the impeller vanes. It occurs in axial, centrifugal shrouded and un-shrouded pumps. It is important to detect this phenomenon at the earliest stage and distinguish it from other undesirable phenomenon such as cavitation.

Hutton [94] investigated the feasibility of detecting AE in the presence of hydraulic noise. It was noted that artificially seeded AE bursts were detected above background operational noise for turbulent flow, with and without cavitations. Furthermore, Hutton noted that the presence of cavitations in the system increased the operational AE noise levels by a factor of 50. In addition, cavitation was found to generate a significant increase in noise level below $500 \mathrm{KHz}$. Hutton placed AE sensors on the pipe. Darling and Johnston [95] that the AE from a high pressure hydraulic pump during cavitation was wide band noise; up to $1 \mathrm{MHz}$. Darling and Johnston noted that during cavitation there was little change in the vibration signature from normal operation which was not the case with AE observations. It was also commented that the position of the AE sensor was insensitive to mounting position whilst the reverse was observed with the vibration senor.

Al-Maskari [96] attempted to detect incipient cavitation with AE but concluded that whilst the inception of cavitation was not detectable with AE, fully developed cavitation was detectable. Another interesting observation by Al-Maskari was the variation in $\mathrm{AE}$ activity at flow rates below the BEP and it was suggested that 
investigations on applying $\mathrm{AE}$ to cavitation detection should be concentrated at the BEP. Al-Maskari placed the AE sensor on the pump casing. Sundt [32] detailed a case study on the application of AE for detecting pump cavitation. It was shown that during cavitation AE levels increased whilst vibration levels dropped. Also, Finley [43] presented an industrial case highlighting the successful application of AE to cavitation detection. Cudina [97] whilst promoting the use of audible acoustics (less than $20 \mathrm{kHz}$ ) for monitoring pumps sites some applications of AE for detecting broad band noise associated with cavitation. Al-Sulti et al [98] noted that the use of power spectrum density of $\mathrm{AE}$ acquired over a range of flow rates was not effective in detecting cavitation. However, it was noted that the use of higher order spectral analysis (bi-coherence) showed improved sensitivity of AE over vibration for early detection of cavitation. The results are in contrast to nearly all published work on AE for monitoring cavitation where a clear increase in AE levels was noted without the need for advanced signal processing.

The papers reviewed above have clearly associated AE with the collapse of cavitation bubbles. The presence of cavitation has been shown to increase operational AE noise levels. Recently Alfayez et al [99, 100] undertook experimental tests on a range of pumps in an attempt to correlate incipient cavitation with AE activity. Results showed a clear relationship between AE activity measured from the pump casing, suction and discharge pipes, and incipient cavitation. At a high NPSH value, when incipient cavitation is known to occur, a significant increase in AE was observed. Experiments were conducted for several flow rates on different sized pumps to validate this assumption. Further reduction in NPSH resulted in decrease in measured AE levels due to the presence of bubble clouds. Observations of the frequency content of 
captured AE time wavefroms showed a shift in frequency range for incipient and developed cavitation. Results of this study also showed that the measurement of AE

r.m.s levels could be employed for determining the BEP of pumps which offers enormous opportunities within the industry. Sikorska and Hodkiewicz [101] reiterated the observations of Alfayez $[99,100]$ noting that $\mathrm{AE}$ was able to detect off duty conditions in double suction pumps. Furthermore Sikorska and Hodkiewicz noted that AE could be used to detect cavitation and recirculation and postulated that low flow AE activity was initiated by recirculation whilst high flow AE activity was due to incipient cavitation. Cavitation is known to occur more easily at higher flow rates [97].

\section{Monitoring engines and rotating structures with Acoustic Emission}

Industries all over the world use various machines and structures to manufacture and distribute various goods and services to global customers. These include rotating and reciprocating machines and mechanical structures of all sizes, shapes and complexities. Damage assessment of these assets (both old and new) is very crucial as it determines the quality, reliability, availability, maintainability and the life expectancy. The reliability and health monitoring of both old and new machineries and structures form the subject of extensive research in many academic institutions, government laboratories, defense research establishments, and industrial organisations worldwide. AE technology is now becoming a widely accepted practice in the field of engine and rotating structural monitoring. 
Holroyd et al [90] illustrated the background to the AE approach and its technological developments, which enabled it to be used as a means of dynamically probing the operation of machineries and mechanisms, and, attempted to clarify the opinions held on the similarities and differences of the $\mathrm{AE}$ and vibration monitoring techniques when applied to machinery condition monitoring [91]. Holroyd et al [92] illustrated with examples some real benefits of using $\mathrm{AE}$ techniques as a highly sensitive, simple to use and cost effective maintenance tool.

Gill et al [93] described how AE techniques could be implemented as a conditionbased maintenance strategy to monitor the inlet and outlet valves of reciprocating compressors. The investigation was based on an 8-cylinder, horizontally opposed, single acting, two-stage compressor used to compress ethylene at a large plastics plant. Gill highlighted the possibility of detecting fluid movement with $\mathrm{AE}$ technology. The sensor required very little space and was non-intrusive, which was a major benefit in the hostile conditions. The results revealed the practical deployment of AE sensors for condition monitoring applications.

Fog et al [94] conducted an experimental investigation into detecting exhaust valve burn-through on a 4-cylinder, 500mm bore, 2-stroke marine diesel engine with an output of approx. 10,000 BHP. The investigation comprised monitoring 3 different valve conditions (normal, leak \& large leak). Vibration \& structure-born stress waves (AE) were monitored. The results showed that the AE signals contained more information for identifying valve and injector related mechanical events during the combustion process than time-series recorded from other sensors. Features of the AE signals were extracted using principal component analysis (PCA). A feed-forward 
neural classifier was used to discriminate between 3 valve conditions. Friis-Hausen et al [95] identified efficient classifiers for detection of two different failure modes in marine diesel engines (exhaust valve leaks and defective injection (misfire)). The purpose of the exhaust valve is to seal the combustion chamber from the surroundings during compression, thus securing maximum pressure in the cylinder during the combustion event. This ensures maximum engine performance in terms of output power. This study identified an efficient classifier, which could discriminate completely between leak sizes in the exhaust valve, based on the recorded r.m.s AE signals. An efficient classifier for detection of misfire was also developed. El-Ghamry et al [96] illustrated the potential of AE sensing to determine the strength of the airfuel mixture in 30.56 litre Perkins 4-stroke, 8-cylinder turbocharged gas engine. AE, acceleration, inside cylinder pressure and timing signals were monitored during the tests. The results revealed that the AE signal showed additional features, which could be used to identify the strength of the gaseous fuel mixture.

Results that showed that indirect measurements of cylinder pressure from diesel engines with AE technology was presented by El-Ghamry [97]. The AE r.m.s was correlated to the pressure in the time and frequency domain. Furthermore, the complex cepstrum analysis was used to model the pressure readings from the complete combustion phase of the engine. El-Ghamry noted the advantage of employing the cepstral analysis for the model, stating that it used the frequency content of the AE r.m.s signal rather than the energy content, which gave the advantage over signals with low energy content. The application of AE technology to modelling the pressure originated from previous studies by El-Ghamry [98]. In the latter investigation El-Ghamry attempted to develop generic techniques for 
diagnosing faults in reciprocating machines. The generic pattern recognition technique developed was based on the time-domain AE r.m.s signals, statistical feature extraction from the time-domain signal and correlation of the AE response to specific events in the engines. Steel et al [99] recently reviewed developments in monitoring engines with $\mathrm{AE}$. It was noted that $\mathrm{AE}$ signals could be associated with the actual operational and degrading processes in the engine. Furthermore this could be accomplished non-intrusively. It was also stated that analysis of AE data could be enhanced with a detailed knowledge of the operating conditions of the engine, such as injector timing, running speed and valve movement.

Mba [100] presented a case study on the application of high frequency acoustic emissions as a means of detecting early stages of loss of mechanical integrity in low speed rotating machinery. Investigations were centered on the Rotating Biological Contactor (RBC) which was used for sewage treatment in small communities and rotates at approximately $1 \mathrm{rpm}$. The results presented were obtained from an operational unit that suffered a fractured stub shaft retaining bolt head. Evidence to support the inadequacies of vibration analysis and the applicability of acoustic emissions to detecting this fault condition were detailed. The results of the case study pointed to the potential of AE for diagnosing serious mechanical defects where vibration analysis would be ineffective. The investigation showed that AE activity could be related to not only the fractured bolt but also to loose bolts. The mechanism for generation $\mathrm{AE}$ signatures was the rubbing in the threaded bolt within its recess and the rubbing and/or crushing of the fractured bolt shank with wear and rust particles within the clearance hole of the stub shaft. A typical AE parameter such as amplitude can provide valuable information on the clamped condition of a component on a low 
speed rotating machine. These observations confirmed the finds of Hanel and Thelen [101, 102, 103] where a relationship between AE activity and the tensile stress on a bolt was established. A direct correlation between increased AE activity and plastic deformation of the bolt was presented. Furthermore, the investigators proposed that the low level AE activity in the elastic range of the bolt corresponded to the friction process in the thread.

Smulders and Loob [104] employed the use of enveloping and high frequency AE technology for monitoring: bearing fault detection; very slow speed bearing; rail car turntables; and lubrication condition in paper mill machinery. Mba et al [105, 106] presented the results of a study into the use of stress wave analysis as a means of detecting early stages of loss of mechanical integrity in low speed rotating machinery. The source of AE was attributed the breakage, and entrapment, of surface asperities as a result of relative movement of clamped components that had lost predefined tightening torques (loose clamped components). Autoregressive (AR) co-efficients associated with each $\mathrm{AE}$ provided an efficient parameter for classification and diagnostics. Holroyd [107] reviewed some of his development work in applying the AET to machinery condition monitoring over the last decade and also introduced new developments in the field of condition monitoring of structures.

\section{Conclusion}

Acoustic Emission Technology is a continuously evolving multidiscipline and is now the focus of intense research and application based studies. The wealth of knowledge discovered, generated and disseminated in this evolving discipline is itself proof of its 
diverse applicability. The interests to develop new technologies to overcome the many hitherto unsolved problems in condition monitoring and diagnostics of complex industrial machinery applications offers immense opportunities for the $\mathrm{AE}$ Technology to grow unabated. This is also reflected by the significant growth in the global demands for the AE sensors. With the accelerating speed in the growth of intelligent information, sensor and data acquisition technologies, combined with the rapid advances in intelligent signal processing techniques, a healthy growth in the application of $\mathrm{AE}$ in many engineering, manufacturing, processing and medical sectors is to be expected. The application of AE in prognosis is yet to be fully explored and exploited. We are still a long way away from interpreting and fully understanding the wonderful 'sounds of $A E$ ' from rotating machines.

\section{References}

1 Pao, Y-H., Gajewski, R.R. and Ceranoglu, A.N. (1979), Acoustic emission and transient waves in an elastic plate, J. Acoust. Soc. Am., 1979, 65(1), 96 102.

2 Pollock AA, Acoustic Emission Inspection, Physical Acoustics Corporation, Technical Report, 1989, TR-103-96-12/89.

3 Mathews, J. R. Acoustic emission, Gordon and Breach Science Publishers Inc., New York. 1983, ISSN 0730-7152.

4 Viktorov. I. A., Rayleigh and Lamb Waves; Physical theory and application, Plenum press, New York,, USA. 1967. 
5 Non-destructive testing handbook, Volume 5 - Acoustic Emissions. (Published by the American Society of Non-destructive testing) ISBN 0931403-02-2.

6 Catlin Jr., J.B. The Use of ultrasonic diagnostic technique to detect rolling element bearing defects. Proceeding of Machinery and Vibration Monitoring and Analysis Meeting, Vibration Institute, USA, April 1983, 123-130.

7 Balerston, H. L., The detection of incipient failure in bearings, Materials Evaluation, Vol 27, 121-128, 1969.

8 Rogers, L. M., The application of vibration analysis and acoustic emission source location to on-line condition monitoring of anti-friction bearings. Tribology International, 1979; 51-59.

9 Yoshioka T, Fujiwara T. New acoustic emission source locating system for the study of rolling contact fatigue, Wear, 81(1), 183-186.

10 Yoshioka T, Fujiwara T. Application of acoustic emission technique to detection of rolling bearing failure, American Society of Mechanical Engineers, Production Engineering Division publication PED, 1984, 14, $55-76$.

11 Hawman, M. W., Galinaitis, W. S, Acoustic Emission monitoring of rolling element bearings, Proceedings of the IEEE, Ultrasonics symposium, 1988, 885-889

12 Holroyd, T.J. and Randall, N., (1993), Use of Acoustic Emission for Machine Condition Monitoring, British Journal of Non-Destructive Testing, 1993, 35(2), 75-78. 
13 Holroyd, T. Condition monitoring of very slowly rotating machinery using AE techniques. 14th International congress on Condition monitoring and Diagnostic engineering management (COMADEM'2001), Manchester, UK, 46 September 2001, 29, ISBN 0080440363

14 Bagnoli, S., Capitani, R. and Citti, P. Comparison of accelerometer and acoustic emission signals as diagnostic tools in assessing bearing. Proceedings of 2nd International Conference on Condition Monitoring, London, UK, May $1988,117-125$.

15 Tandon, N. and Nakra, B.C, Defect Detection of Rolling Element Bearings by Acoustic Emission Method, Journal of Acoustic Emission, 1990; 9(1) 25-28.

16 Choundhury, A. and Tandon, N., Application of acoustic emission technique for the detection of defects in rolling element bearings, Tribology International, 2000; 33, 39-45

17 Tan, C.C. Application of acoustic emission to the detection of bearing failures. The Institution of Engineers Australia, Tribology conference, Brisbane, 3-5 December 1990, 110-114.

18 Huguet, S, Godin, N, Gaertner, R, Salmon, L, Villard, D. Use of acoustic emission to identify damage modes in glass fibre reinforced polyester. Composites Science and Technology, 2002, 62, 1433-1444.

19 Yoshioka, T., korenaga, A., Mano, H. and Yamamoto, T. Diagnosis of rolling bearing by measuring time interval of Acoustic Emission generation. Journal of Tribology, Transactions of ASME, Vol. 121, July 1999, 468-472.

20 Morhain, A, Mba, D, Bearing defect diagnosis and acoustic emission, Journal of Engineering Tribology, I Mech E, Vol 217, No. 4, Part J, p 257-272, 2003. ISSN 1350-6501. 
21 Kakishima, H., Nagatomo, T., Ikeda, H., Yoshioka,, T., Korenaga, A., Measurement of Acoustic Emisión and vibration of rolling bearings with an artificial defect, QR of RTRI, Vol 41, No. 3,127-130, 2000.

22 Kaewkongka, T and Au, Y. H. J., application of Acoustic Emission to condition monitoring of rolling element bearings. Measurement and Control, Vol 34, no. 8, 254-247, 2001.

23 Schoess, J. N.; Development and application of stress-wave acoustic diagnostics for roller bearings, Proceedings of SPIE, The International society for optical engineering, Vol 3986, 58-70, 2000.

24 Price, E. D., Lees, A. W., M.I. Friswell, Application of high frequency monitoring for classification of rolling element bearing failures. Key Engineering Materials, Vol 204-205, 173-182, 2001

25 Shiroishi, J., Li, Y., Liang, L. S., Kurfess, T., Danyluk, S., Bearing condition via vibration and acoustics emission measurements. Mechanical Systems and Signal Processing, Vol. 11, no. 5, 1997, 693-705.

26 Hoeprich, M. R., Rolling Element bearing fatigue damage propagation, Transactions of ASME, Journal of Tribology, Vol. 114, April 1992, 328-333.

27 Abdullah M. Al-Ghamdi, P. Cole, Rafael Such, D. Mba, Estimation of bearing defect size with Acoustic Emission, INSIGHT, Vol. 46, no. 12, 758-761, Dec 2004.

28 Abdullah M. Al-Ghamdi and D. Mba. A comparative experimental study on the use of Acoustic Emission and vibration analysis for bearing defect identification and estimation of defect size. Mechanical Systems and Signal Processing, Accepted MSSP04-98R2. 2005. 
29 Li, Y., Billington, S., Zhang, C., Kurfess, T., Danyluk, S., Dynamic prognostic predicition of defect propagation on rolling element bearings. ASME/STLE Tribology conference, Toronto, Ontario, Canada, Oct 26-28, 1998, 385-392.

30 Vibha Bansal, Gupta, B.C, Arun Prakash, Eshwar, V. A, Quality inspection of rolling element bearing using acoustic emission technique, 1990, Journal of Acoustic emission, 9(2), 142-146.

31 Li, C. J, Li, S. Y., Acoustic Emission analysis for bearing condition monitoring, Wear, 185, 1985, 67-74.

32 Sundt, P. C, Monitoring Acoustic Emission to detect mechanical defects, InTech, December 1979, 43 - 44

33 Barclay, J., Bannach, R, Multi-parameter condition monitoring, Noise and vibration worldwide, March 1992, 17 - 20.

34 Badi, M.N.M., Johnson, D.E. \& Trmal, G.J. (1990). The Use of Stress Wave Sensors for the Diagnosis of Bearing Faults. Proceedings of COMADEM 1990. Chapman \& Hall, London. 20-25. ISBN 0 - 412 - 38560 - 0

35 Sturm, A., Ellmer, G. \& Billhardt, S. (1992). Analysis of High-Frequency Acoustic Signals of Machines with Rolling-Element Bearings and Sliding Bearings. Ibid. 184-190.

36 Javed, M. \& Littlefair, G. (1993). Neural Networks based System for Rotating Machinery. Proceedings of COMADEM 1993. University of West of England, Bristol. 418-23. ISBN 1 - 871056 - 72 - 1

37 Neill, G.D., Benzie, S., Gill, J.D., Sandford, P.M., Brown, E.R., Steel, J.A. \& Reuben, R.L. (1998). The Relative Merits of Acoustic Emission \& Acceleration Monitoring for the Detection of Bearing Defects. Proceedings of COMADEM 1998.Monash University, Australia. 651-659. 
38 Salvan, S.M.E., Parkin, R.M., Coy, J. \& Li,W. (2001). Intelligent Condition Monitoring of Bearings in Mail Processing Machines using Acoustic Emission. Proceedings of COMADEM 2001. Elsevier, Oxford. 67-74. ISBN $0-08-044036-3$

39 Parikka, R., Vidqvist, V., Vaajoensuu, E. \& Tervo, J. (2002). Test Equipment \& Diagnostic System for Determining Lubrication Condition \& Running Clearance of Rolling Bearings. Proceedings of COMADEM 2002. COMADEM International, Birmingham. 153-162. ISBN 0 - 9541307 - 0 - 7

40 Morhain, A. \& Mba, D. (2002). Defect Diagnosis of Lightly Loaded Bearings with Acoustic Emissions. Proceedings of COMADEM 2002. COMADEM International, Birmingham. 492-502. ISBN 0 - 9541307 - 0 - 7.

41 Yibo Edward Fan, Fengshou Gu and Andrew Ball, Condition monitoring of rolling element bearings using advanced acoustic emission signal analysis technique, $18^{\text {th }}$ International Congress and Exhibition on Condition Monitoring and Diagnostic Engineering Management (COMADEM 2005), Cranfield, UK, August 31 $1^{\text {st }}-2^{\text {nd }}$ September 2005.

42 Holroyd, T. J., Acoustic Emission as a basis for the condition monitoring of industrial machinery. Proceedings of the $18^{\text {th }}$ Machinery vibration seminar, Canadian Machinery vibration association, 27-29 April 2000, Halifax, Nova Scotia, Canada, p 125-131. ISBN 1-55131-045-7.

43 Finley, R. W, Computerisation preventive maintenance systems using modified Acoustic Emission techniques, Materials Evaluation, 38(8), p 15-20, August 1980.

44 Jamaludin, N., Jailani, M. \& Nor, M. (2002). Lubrication Monitoring in a Low Speed Bearing Using Acoustic Emission Technique. Proceedings of 
COMADEM 2002. COMADEM International, Birmingham. 521-527. ISBN 0 $-9541307-0-7$

45 Miettinen, J. \& Salmenperä, P. (2000). Acoustic Emission Monitoring of Grease Lubricated Rolling Bearings. Proceedings of COMADEM 2000. MFPT Society, USA. 21-30. ISBN 0 - $9635450-2$ - 7

46 Miettinen J, Andersson, P. Acoustic emission of rolling bearings lubricated with contaminated grease, Tribology International, 2000, 33(11), 743-802 .

47 Berry, J. E., 1992 Required vibration analysis techniques and instrumentation on low speed machines ( particularly 30 to 300 RPM machinery ), Technical Associates of Charlotte Inc.,Advanced Vibration Diagnostic and Reduction Techniques.

48 Canada, R.G., and Robinson, J.C., 1995 Vibration measurements on slow speed machinery. Predictive Maintenance Technology National Conference (P/PM Technology), pp 33-37, Vol. 8, no. 6. Indianapolis, Indiana.

49 Murphy, T.J., 1992 The development of a data collector for low-speed machinery. 4th international Conference on Profitable Condition Monitoring, bH ${ }^{\mathrm{r}}$ Group Ltd., pp 251-258, 8-10 Dec., Stratford-upon-Avon, UK.

50 Robinson, J.C, Canada, R.G., and Piety, R.G. 1996 Vibration Monitoring on Slow speed Machinery: New Methodologies covering Machinery from 0.5 to 600rpm. Proc. 5th International Conference on Profitable Condition Monitoring - Fluids and Machinery Performance Monitoring, pp 169-182, bH ${ }^{\mathrm{r}}$ Group Ltd., Publication 22, Harrogate, UK.

51 Kuboyama, K., Development of Low Speed Bearing Diagnosis Technique, NKK Fukuyama Works, Fukuyama City, Hiroshima, Japan. 
52 McFadden, P. D., and Smith, J. D. Acoustic emission tranducers for the vibration monitoring of bearings at low speeds, Report no. CUED/CMech/TR29 1983.

53 Smith, J.D. 1982 Vibration monitoring of bearings at low speeds, Tribiology International, pp 139-144.

54 Tavakoli, M. S., Review of bearing monitoring - Application of Acoustic Emission, $1^{\text {st }}$ International conference on Acoustic Emission in Manufacturing; Edited by Vahaviolos, S. J., 16 - 19 Sept, 1991, Boston, Massachusetts, USA.

55 Holroyd, T.J. (1993). Acoustic Emission Monitoring of Slow Moving Bearings. Proceedings of COMADEM 1993. University of West of England, Bristol. 518. ISBN 1 - $871056-72$ - 1

56 Miettinen, J. \& Pataniitty, P. (1999). Acoustic Emission in Monitoring Extremely Slowly Rotating Rolling Bearing. Proceedings of COMADEM 1999. Coxmoor Publishing Co, UK. 289-297. ISBN 1 - 901892 - 13 - 1

57 N. Jamaludin, Dr. D. Mba, Dr. R. H. Bannister Condition monitoring of slowspeed rolling element bearings using stress waves. Journal of Process Mechanical Engineering, Pro. Inst. Mech Eng., 2001, 215(E):, Issue E4, 245271.

58 Sato, I. Rotating machinery diagnosis with acoustic emission techniques, Electrical engineering in Japan, 1990; 10(2):115-127.

59 Sturm A, Uhlemann, S., Diagnosis of plain bearings by Acoustic Emission analysis, Measurements, 3(4), 185-191, 1985. 
60 Willaims, T., Ribadeneira, X., Billington, S. and Kurfess, T., Rolling element bearing diagnosis in run-to-failure lifetime testing. Mechanical Systems and Signal Processing, Vol 15, no. 5, 2001, 979-993.

61 Miyachika, K., Oda, S. and Koide, T. Acoustic Emission of bending fatigue process of spur gear teeth. Journal of Acoustic Emission. 13(1/2). S47-S53. 1995.

62 Miyachika, K., Zheng, Y., Tsubokura, K., Oda, S., Kanayama, Y., Koide, T., Namba, C. and Hayashi, T. Acoustic Emission of bending fatigue process of super-carburised spur gear teeth. Progress in Acoustic Emission XI. The Japanese Society for NDI. 304-310. 2002.

63 Wheitner, J., Houser, D. and Blazakis, C. Gear tooth bending fatigue crack detection by Acoustic Emissions and tooth compliance. ASME technical paper 93FTM9. 1-7. 1993.

64 Singh, A., Houser, D. R., and Vijayakar, S. Detecting Gear Tooth Breakage Using Acoustic Emission: A Feasibility and Sensor Placement Study. Journal of Mechanical Design. 121. 587-593. 1999.

65 Siores, E. and Negro, A.A. Condition Monitoring of a Gear Box Using Acoustic Emission Testing. Material Evaluation. 183-187. 1997.

66 Singh, A., Houser, D. R., and Vijayakar, S. Early Detection of Gear Pitting. Power Transmission and Gearing Conference, ASME. DE-Vol. 88, 673-678. 1996.

67 Raad, A., Zhang, F., Randall, B. and Sidahmed, M. On the comparison of the use of AE and vibration analysis for early gear fault detection. The $8^{\text {th }}$ Western Pacific Acoustics Conference. Melbourne, Australia. 7-9 April 2003. 
68 Sentoku, H. AE in Tooth Surface Failure Process of Spur Gears. Progress in Acoustic Emission IX. AEWG \& AE Group. S19-S24. 1998.

69 Badi, M.N.M., Engin, S.N. and Schonfeld, D. Fault classification of a model drive-line using time domain data. COMADEM 1996. Sheffield, UK. 43-50. 1996. ISBN $1-85075-635$ - X

70 Tandon, N. and Mata, S. Detection of Defects in Gears by Acoustic Emission Measurements. Journal of Acoustic Emission. 17(1-2). 23-27. 1999.

71 Al-Balushi, K. R. and Samanta, B. Gear Faults Diagnosis Using Energy-Based Features of Acoustic Emission. Journal of Systems and Control Engineering. 216. 249-263. 2002.

72 Al-Balushi, K.R. \& Samanta, B. (2000). Gear Fault Diagnostics using Wavelets and Artificial Neural Networks. COMADEM 2000. 1001-1010. 0 $9635450-2-7$

73 Tan, C. K and Mba, D., Identification of the Acoustic Emission source during a comparative study on diagnosis of a spur gearbox, Tribology International, Volume 38, Issue 5, Pages 469-480, May 2005.

74 Tan, C. K and Mba, D, Limitation of Acoustic Emission for identifying seeded defects in gearboxes. Journal of Non-Destructive Evaluation, Vol. 24, Issue 2, June 2005.

75 Toutountzakis, T. and Mba, D. Observation of Acoustic Emission Activity During Gear Defect Diagnosis. NDT and E International. 36(7). 471-477. 2003.

76 Price, E. D., Lees, A. W., Friswell, M. I., Detection of severe sliding and pitting fatigue wear regimes through the use of broadband acoustic emission. 
Proceedings of the I MECH E Part J Journal of Engineering Tribology, 219 (2), 85-98, 2005.

77 Tan, C. K., Phil Irving, David Mba, A comparative experimental study on the diagnostic and prognostic capabilities of Acoustics Emission, Vibration and Spectrometric Oil Analysis for spur gears. Mechanical Systems and Signal Processing, Submitted and under review, Jan 2005.

78 Tan, C.K., P. Irving and D. Mba, Prognostic potential of the Acoustic Emission technique for spur gears, 'Essential technologies for successful prognostics', Proceedings of the $59^{\text {th }}$ Meeting of the Society for Machinery Failure Prevention Technology (MFPT), Virginia Beach, Virginia, USA, April 18-21, 2005, p67-76.

79 Tan, C. K., Phil Irving and David Mba, Opportunities offered by acoustic emission (AE) for prognosis of spur gears, Proceedings of the $17^{\text {th }}$ International congress on Condition Monitoring and Diagnostic Engineering management, COMADEM, p 152-163, Aug 2004. ISBN 0-954 1307-1-5.

80 Chee Keong Tan, Phil Irving and David Mba, Diagnostics and prognostics with Acoustic Emission, Vibration and Spectrometric Oil Analysis for spur gears; a comparative study, INSIGHT, Vol. 47 No. 8, August 2005

81 Tan, C. K and Mba, D, Acoustic emission source at the gear mesh, Proceedings of the $17^{\text {th }}$ International congress on Condition Monitoring and Diagnostic Engineering management, COMADEM, p 145-151, Aug 2004. ISBN 0-954 1307-1-5.

82 Tan, C. K and Mba, D, The source of Acoustic Emission during meshing of spur gears, The $26^{\text {th }}$ European conference on Acoustic Emission testing, EWGAE, Sept 15 - 17, 2004, p 559-565. ISBN 3-931381-58-7 
$83 \mathrm{C} \mathrm{K}$ Tan and D Mba, Correlation between Acoustic Emission activity and asperity contact during meshing of spur gears under partial elastohydrodynamic lubrication. Accepetd, TRIBOLOGY LETTERS, 2005

84 P. J. McNulty and I.S. Pearsall, Cavitation inception in pumps, Journal of Fluids Engineering, Vol. 104, p 99 - 104, March 1962.

85 McNulty P.J, W M Deeprose, Scaling of Non-Cavitating and Cavitating Pump Noise Acoustic for Pumps, 1978. Risley, UK. paper 3, 1-17. ISBN 10185621

86 P. J. McNulty (1981) Measurement Techniques and Analysis of Fluid-Borne Noise in Pumps. National Engineering Laboratory. NEL Report No 674.

87 Derakhshan, O., J. Rechard Houghton, R. Keith Jones (1989). Cavitation Monitoring of Hydroturbines with RMS Acoustic Emission Measurements. World Meeting on Acoustic Emission , p305-315, March 1989.

88 Guelich, J. F and Bolleter, U., Pressure pulsations in centrifugal pumps, Journal of Vibration and Acoustics, Transactions of the ASME, Vol. 114, p 272-279, April 1992.

89 Fraser, W. H., Flow recirculation in centrifugal pumps, Proceedings of the $10^{\text {th }}$ annual turbomachinery symposium, Turbomachinery laboratory, Texas, USA., p 95-100, 1981.

90 G D Neill, R L Reuben, P M Sandford (1997). Detection of Incipient cavitation in Pumps Using Acoustic Emission. Journal of Process Mechanical Engineering [H.W. Wilson - AST]. 1997. Vol. 211, E4; p. 267 )

91 G D Neill, R L Reuben, P M Sandford, E.R Brown, J. A. Steel. Detection of Incipient cavitation in Pumps Using Acoustic Emission. In proceedings of COMADEM 96. Sheffield University, 1996, 391-401. ISBN 1-85075-635-X 
92 Neill, G.D., Reuben, R.L., Sandford, P.M., Brown, E.R. and Steel, J.A. (1996). Detection of Incipient Cavitation in Pumps using Acoustic Emission. Proceedings of COMADEM 1996. Sheffield Academic Press, Sheffield. 391$401.1-85075-635-X$

93 Neill, G.D., Brown, E.R., Reuben, R.L., Sandford, P.M.and Steel, J.A. (1998). Detection of Recirculation in Pumps using Acoustic Emission. COMADEM 1998.Monash University, Australia. 643-649. 0 - 7326 - 2027 - 9

94 P. H. Hutton (1969). Detecting Acoustic Emission in Presence of Hydraulic Noise. Non Destrictive testing May 1969.

95 Darling, J. and Johnston, D. N, The use of Acoustic Emission for condition monitoring in high-pressure hydraulic pumps, paper C414/011, EUROTECH Direct 91, IMechE, 2-4 July, 1991, Birmingham, UK. P 137-143.

96 A. Almaskari (1984). Detecting of cavitation in centrifugal pump using acoustic emission. MSc. Thesis, Cranfield University.

97 Cudina, M., detection of cavitation phenomenon in a centrifugal pump using audible sound, Mechanical Systems and Signal Processing, 17(6), 1335-1347, 2003.

98 S. Al-Sulti, B. Samanta, K. R. Al-Balushi, M. Al-Zedjali, S. A. Al-Araimi, and R. A. Siddiqi, Comparison of signal processing techniques for detecting pump cavitation inception, $18^{\text {th }}$ International Congress and Exhibition on Condition Monitoring and Diagnostic Engineering Management (COMADEM 2005), Cranfield, UK, August $31^{\text {st }}-2^{\text {nd }}$ September 2005.

99 L. Alfayez, D. Mba, G. Dyson The application of Acoustic Emission for detecting incipient cavitation and the best efficiency point of a $60 \mathrm{KW}$ 
centrifugal pump; case study. NDT \& E International, Vol 38, no. 5, 354-358. 2005.

100 L. Alfayez, D. Mba, Detection of incipient cavitation and determination of the best efficiency point for centrifugal pumps using Acoustic Emission. Journal of Mechanical Process Engineering, Part E, IMechE, 2005.

101 J. Z. Sikorska and M.Hodkiewicz, Comparison of acoustic emission, vibration and dynamic pressure measurements for detecting change in flow conditions on a centrifugal pump. $18^{\text {th }}$ International Congress and Exhibition on Condition Monitoring and Diagnostic Engineering Management (COMADEM 2005), Cranfield, UK, August $31^{\text {st }}-2^{\text {nd }}$ September 2005.

102 Holroyd, T.J., Randall, N. \& Lin, D. (1996). Acoustic Emission as a Means of Dynamically Probing the Operation of Machines \& Mechanisms. COMADEM 1996. 529-536. $1-85075-635-X$

103 Holroyd, T.J. \& Brashaw, C. (1999). A Critical Appraisal of Monitoring Elastic Waves as a Means of Detecting \& Diagnosing Machine Faults. Proceedings of COMADEM 1999. Coxmoor Publishing Co. UK. 491-497. 1 $901892-13-1$

104 Holroyd, T.J. \& Randall, N. (1993). Field Application of Acoustic Emission to Machinery Condition Monitoring. Proceedings of COMADEM 1993. University of West of England, Bristol. 217-222. 1 - 871056 - 72 - 1

105 Gill, J.D., Brown, E.R., Twite, M., Horner, G., Reuben, R.L. Steel, J.A. (1998). Monitoring of a Large Reciprocating Compressor. Proceedings of COMADEM 1998. Monash University, Australia. 317-326. 0 - 7326 - 2027-9 
106 Fog, T.L, Brown, E.R., Hanson, H.S., Madsen, L.B., Sørensen, P., Hansel, E.R., Steel, J.A., Reuben, R.L and Pedersen, P.S (1998). Proceedings of COMADEM 1998. Monash University, Australia. 269-278. 0 - 7326 - 2027-9 107 Friis-Hausen, A. and Fog, T.L. (2001). Monitoring Exhaust Valve Leaks and Misfire in Marine Diesel Engines. Proceedings of COMADEM 2001. Elsevier, Oxford. 641-648. 0 - $08-044036$ - 3

108 El-Ghamry, M.H., Brown, E., Ferguson, I.G., Gill, J.D., Reuben, R.L., Steel, J.A., Scaife, M. and Middleton, S. (1998). Gaseous Air-Fuel Quality Identification for a Spark Ignition Gas Engine using Acoustic Emission Analysis. Proceedings of COMADEM 1998. Monash University, Australia. 235-244. $0-7326-2027-9$

109 El-Ghamry, M., Steel, J.A.; Reuben, R.L.; Fog, T.L., Indirect measurement of cylinder pressure from diesel engines using acoustic emission, Mechanical Systems and Signal Processing, v 19(4),2005, 751-765.

110 El-Ghamry, M.H., Reuben, R.L.; Steel, J.A., The development of automated pattern recognition and statistical feature isolation techniques for the diagnosis of reciprocating machinery faults using acoustic emission, Mechanical Systems and Signal Processing, 17(4), 2003, 805-823.

111 Steel, J.A. Reuben, R.L., Recent developments in monitoring of engines using acoustic emission, Journal of Strain Analysis for Engineering Design, 40(1), 2005, 45-57.

112 Mba, D., Applicability of acoustic emissions to monitoring the mechanical integrity of bolted structures in low speed rotating machinery: case study. NDT and E International. Vol. 35, No. 5, pp. 293-300. 2002 
113 Hanel, V.; Thelen, W. Monitoring screws under tensile load using acoustic emission analysis. Instrumentation and Measurement, IEEE Transactions, April 1996 ; 45(2): 547 -550.

114 Hanel, V.; Thelen, W. Acoustic Emission testing of bolted connections under tensile stress. $22^{\text {nd }}$ European conference on Acoustic Emission Testing. The Robert Gordon University, Aberdeen. 29-31 May 1996;p 185-188.

115 Hanel,V.;Thelen,W. Determination of load condition of standard screws during tensile tests using Acoustic Emission analysis. Instrumentation and Measurement Technology Conference, 1995. IMTC/95. Proceedings. Integrating Intelligent Instrumentation and Control, IEEE 1995; p284.

116 Smulders, A. and Loob, C. (1994). Machine Condition Monitoring using Multi-Parameter Measurements. Proceedings of COMADEM 1994. Tata McGraw-Hill Publishing Co. India. 147- 152. 0 - 07 - 462360 - 5

117 Mba, D.U., Bannister, R.H., Leslie, J.T. \& Findlay, G.E. (1996). Condition Monitoring of Low Speed Rotating Machinery. Proceedings of COMADEM 1996. Sheffield Academic Press, Sheffield. 73-84. 1 - 85075 - 635 - X

118 Mba, D. \& Hall, L. (2001). Monitoring the Integrity of Low Speed Rotating Machines. COMADEM 2001. 697-707.0 - 08 - 04436 - 3

119 Holroyd, T.J. (2002). Simplifying the Condition Monitoring of Machines \& Structures using Acoustic Emission. Proceedings of COMADEM 2002. COMADEM International, Birmingham. 180-188. 0 - 9541307 - 0 - 7. 